\title{
Nevus Comedonicus With Acne Vulgaris and Obesity
}

\author{
Caterina Mazzella, ${ }^{1}$ Gabriella Fabbrocini, ${ }^{1}$ Maria Antonietta Luciano, ${ }^{2}$ Sara Cacciapuoti $^{1}$
}

\begin{abstract}
1 Dermatology Unit, Department of Clinical Medicine and Surgery, Federico II University of Naples, Italy
2 Dermatology Unit, Department of Public Health, Federico II University of Naples, Italy
\end{abstract}

Key words: nevus, acne, obesity, hamartoma, pilosebaceous unit

Citation: Mazzella C, Fabbrocini G, Luciano MA, Cacciapuoti S. Nevus comedonicus with acne vulgaris and obesity. Dermatol Pract Concept. 2020;10(1):e2020006. DOI: https://doi.org/10.5826/dpc.1001a06

Accepted: September 1, 2019; Published: December 31, 2019

Copyright: (92019 Mazzella et al. This is an open-access article distributed under the terms of the Creative Commons Attribution License, which permits unrestricted use, distribution, and reproduction in any medium, provided the original author and source are credited.

Funding: None.

Competing interests: The authors have no conflicts of interest to disclose.

Authorship: All authors have contributed significantly to this publication.

Corresponding author: Caterina Mazzella, MD, via S Pansini, N. 5, 80131, Naples, Italy. Email: caterinamazzella@libero.it

\section{Introduction}

Nevus comedonicus is a rare hamartoma of the pilosebaceous unit, first described by Kofmann in 1895 [1]. In most cases it is present at birth but may also develop later, usually before the age of 10 years, without racial or sexual preponderance. When appearing in adults, although rare, it is frequently associated with irritation or trauma [2].

The lesions typically present a linear or zosteriform distribution, but extensive areas have already been described, including variant with bilateral involvement. The most frequently affected sites are the face, trunk, neck, and upper limbs. It may affect areas without hair follicles, such as the palms, soles, and the glans penis. Scalp involvement is rare [3]. There may be associations with other skin lesions or abnormalities of the central nervous, musculoskeletal, and ocular system [4].

We present a case of nevus comedonicus in a woman suffering from obesity and acne vulgaris.

\section{Case Presentation}

A 40-year-old woman with a history of obesity and acne presented with an asymptomatic lesion that had appeared 20 years previously on the right thigh. The clinical aspect and extent of the lesion caused relevant psychosocial repercussions, interfering with her personal relationships. At dermatological examination, bundles of dilated hair follicles were observed, filled with dark-colored plugs, presenting a linear distribution in the right thigh with alteration of the skin pigmentation at the site of the lesion; at dermoscopy, well-defined homogeneous areas in dark brown shades were noted (Figure 1, A and B). Some cysts had been treated in the past with antibiotics, leaving some hyperchromic signs on the thigh. The patient also suffered from facial acne (Figure 2) that was resistant to numerous treatments performed in previous years: topical and systemic antibiotics and topical and systemic retinoids. The histological examination performed with a 5-mm diameter punch showed a large group of dilated follicular ostia devoid of hair shafts but filled with keratin layers (Figure 3).

On the basis of the history and typical features of the lesion and histology, a nevus comedonicus in a patient with resistant acne and obesity was diagnosed. Consultations with other specialists excluded the presence of extracutaneous signs or association with genetic syndromes and other diseases or nevus comedonicus syndrome. The patient refused genetic analysis. Because of the extent of the lesion and the possibility of its turning into an unaesthetic scar, we treated 

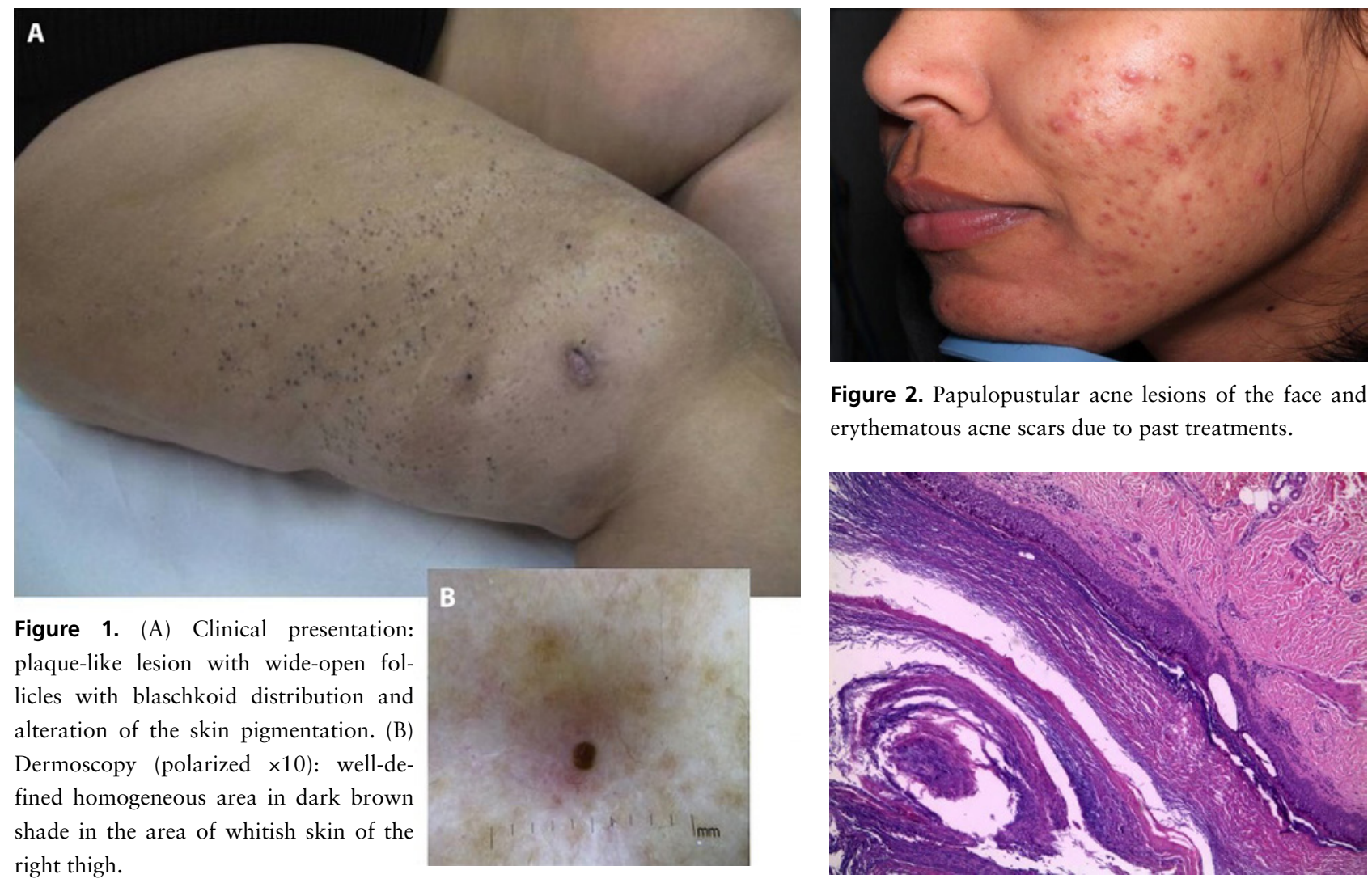

Figure 2. Papulopustular acne lesions of the face and erythematous acne scars due to past treatments.

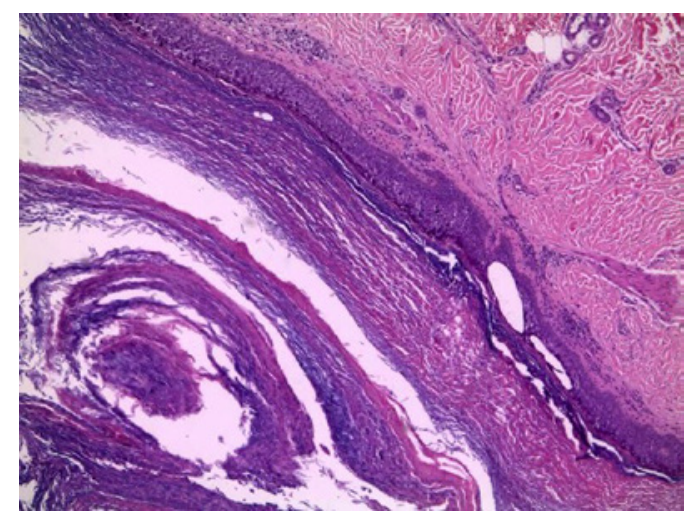

Figure 3. Histology presentation: dilated follicular ostia devoid of hair filled with keratin layers.

the patient with $33 \%$ salicylic acid peeling and home-based topical retinoid therapy, obtaining only partial benefit.

\section{Discussion}

The prevalence of nevus comedonicus has been estimated from 1 in 45,000 to 1 in 100,000. Nevus comedonicus lesions might present with various patterns of distribution: unilateral, bilateral, linear, interrupted, segmental, or blaschkoid [5]. The major histopathological features are large-grouped, dilated follicular ostia devoid of hair shafts but filled with keratin layers. At some locations in the bases of the follicular invaginations one may observe singular rudimentary glands, which are not, however, obligatory as they may be absent. Small cysts, cystic invaginations, and occasionally large cysts may be seen in histopathological investigation; the variable cystic structures are lined by keratinizing squamous epithelium. Hyperkeratosis and acanthosis of the epidermis may be present but not para- or dyskeratosis [6].

Nevus comedonicus syndrome (ORPHA:64754) belongs to the group of epidermal nevus syndromes including Schimmelpenning syndrome, phacomatosis pigmentokeratotica, angora hair nevus syndrome, and Becker nevus syndrome among others in which a genetic basis has not yet been identified [7].
In each case of nevus comedonicus, it is obligatory to rule out the comedonicus syndrome, which may include ocular lesions (cataract, corneal erosion), skeletal abnormalities (syndactyly, clinodactyly, the absence of hand bones on x-ray, scoliosis, vertebral defects), and neurological disturbances (microcephaly, mental deficiency, the dysgenesis of the corpus callosum) [7].

In some cases of nevus comedonicus, a somatic mutation in fibroblast growth factor-receptor gene 2 (FGFR2) has been identified, namely the Ser252Trp missense mutation. FGFR2 is expressed in keratinocytes, hair follicles, and sebaceous glands and has been implicated in induction of hypercornification and comedogenesis. Germline FGFR2 mutations are also associated with acne, as seen in dominant Apert syndrome. When occurring as a mosaic, the acne lesions follow the lines of Blaschko, the pattern of embryological cell development and proliferation [8]. In a recent case, a postzygotic mosaicism was found in exon 4 of FGFR2 (c.758C>G, p.Pro253Arg) in low copy number. A similar mosaicism was also found in 2 other patients in p.Ser252Trp of exon 4 [9].

FGF signaling pathways lead to the interplay of the mTORC1 and FoxO1 pathways; the same pathway is regulated by increased caloric intake, leading to the onset of acne and metabolic syndrome [10]. The 2 pathways could be strictly dependent, explaining our clinical case. 


\section{Conclusions}

We suggest that the coexistence of comedonic nevus, acne, and obesity may not be a random association. Future studies should investigate a screening of phenotypically similar patients for FGFR2 mutations and a possible therapeutic modulation of FGFR signaling.

\section{References}

1. Guldbakke KK, Khachemoune A, Deng A, Sina B. Naevus comedonicus: a spectrum of body involvement. Clin Exp Dermatol. 2007;32(5):488-492.

2. Tchernev G, Ananiev J, Semkova K, Dourmishev LA, Schönlebe J, Wollina U. Nevus comedonicus: an updated review. Dermatol Ther (Heidelb). 2013;3(1) :33-40.

3. Garcia LC, Cabeda LF, Garcia R, Borile G. Nevus comedonicus: surgical treatment. Rev Bras Cir Plast São Paulo. 2000;15:7-14.

4. Yadav P, Mendiratta V, Rana S, Chander R. Nevus comedonicus syndrome. Indian J Dermatol. 2015;60(4):421.
5. Happle R. The group of epidermal nevus syndromes, part I: well defined phenotypes. J Am Acad Dermatol. 2010;63:1-22.

6. Jeong HS, Lee HK, Lee SH, Kim HS, Yi SY. Multiple large cysts arising from nevus comedonicus. Arch Plast Surg. 2012;39(1):6366.

7. Vidaurri-de la Cruz H, Tamayo-Sánchez L, Durán-McKinster C, de la Luz Orozco-Covarrubias M, Ruiz-Maldonado R. Epidermal nevus syndromes: clinical findings in 35 patients. Pediatr Dermatol. 2004;21(4):432-439.

8. Munro CS, Wilkie AO. Epidermal mosaicism producing localised acne: somatic mutation in FGFR2. Lancet. 1998;352(9129):704705 .

9. Melnik B, Vakilzadeh F, Aslanidis C, Schmitz G. Unilateral segmental acneiform naevus: a model disorder towards understanding fibroblast growth factor receptor 2 function in acne? $\mathrm{Br}$ J Dermatol. 2008;158(6):1397-1399.

10. Clatici VG, Voicu C, Voaides C, Roseanu A, Icriverzi M, Jurcoane $\mathrm{S}$. Diseases of civilization-cancer, diabetes, obesity and acne-the implication of milk, IGF-1 and mTORC1. Maedica (Buchar). 2018;13(4):273-281. 